Pacific Journal of Mathematic 


\title{
EQUILIBRIA OF CONTINUOUS TWO-PERSON GAMES
}

\author{
T. Parthasarathy and T. E. S. Raghavan
}

Some sufficient conditions are given to show the existence of equilibrium points with finite spectrum for nonzero-sum two-person continuous games on the unit square. We also examine the question of uniqueness of the equilibrium point for such games.

1. Players I and II choose secretly an $x$ and a $y$ in the closed interval $[0,1]$. Player I receives $K_{1}(x, y)$ and player II receives $K_{2}(x, y)$ where $K_{1}, K_{2}$ are continuous on the unit square. The following theorem is classical in game theory: ([3], see page 156).

There exists a pair of probability distributions $\left(F^{0}, G^{0}\right)$, called Nash equilibrium points satisfying

$$
K_{1}\left(F^{0}, G^{0}\right) \geqq K_{1}\left(x, G^{0}\right) \text { for all } x \text { in } 0 \leqq x<1
$$

and

$$
K_{2}\left(F^{0}, G^{0}\right) \geqq K_{2}\left(F^{0}, y\right) \text { for all } y \text { in } 0 \leqq y \leqq 1
$$

where $K_{1}(F, G)=\iint K_{1}(x, y) d F(x) d G(y)$ and

$$
K_{1}(x, G)=\int K_{1}(x, y) d G(y) \text { etc. }
$$

Let $\mathscr{E}$ be the set of such pairs $\left(F^{0}, G^{0}\right)$.

One can ask the following questions. When does an $\left(F^{0}, G^{0}\right) \in \mathscr{E}$ with the spectrums of $F^{0}$ and $G^{0}$ being finite? For what class of games, $\mathscr{E}$ has a unique point? Here we try to answer these questions by giving some sufficient conditions.

2. In this section we prove the following results.

THEOREM 1. Let $K_{1}(x, y)$ and $K_{2}(x, y)$ be continuous on $0 \leqq x$, $y \leqq 1$. Let $K_{2}(x, y)$ be concave in $y$ for each $x$. Then there is an equilibrium $\left(F^{0}, G^{0}\right)$ such that $G^{0}$ is a degenerate probability distribution and $F^{0}$ is concentrated at most at two points.

THEOREM 2. Let $K_{1}(x, y)$ and $K_{2}(x, y)$ be continuous on $0 \leqq x$, $y \leqq 1$. Let $\left(\partial^{n} / \partial y^{n}\right) K_{2}(x, y) \leqq 0$. Then there is an $\left(F^{0}, G^{0}\right) \in \mathscr{E}$ with the spectrum of $F^{0}$ and $G^{0}$ finite. 
We need the following theorem of Bohnenblust, Karlin, and Shapley [1] in the sequel.

Proposition. Let $K$ be compact convex in $R^{n}$. Let $g_{\alpha}$ be a family of continuous convex functions on $K$. Let $\sup _{\alpha} g_{\alpha}(x)>0$ for each $x \in K$. Then there exists $(n+1)$ induces $\alpha_{1}, \alpha_{2}, \cdots, \alpha_{n+1}$ such that $\sum_{i=1}^{n+1} \lambda_{2} g_{\alpha_{i}}(x)>0$ for all $x$. Here $\left(\lambda_{1}, \lambda_{2}, \cdots, \lambda_{n+1}\right)$ is a probability vector.

REMARK. Theorems 1 and 2 are known for zero-sum games [1], [2]. Our proof follows similar lines.

Proof of Theorem 1. We first prove the result when $K_{2}(x, y)$ is strictly concave in $y$ for each $x$.

Let $\left(F^{0}, G^{0}\right) \in \mathscr{E}$. Since $K_{2}(x, y)$ is strictly concave in $y, \sigma\left(G^{0}\right)$, the spectrum of $G^{0}$ contains just one element, say $y^{0}$. Let $C=\sigma\left(F^{0}\right)=$ the spectrum of $F^{0}$. Consider

$$
\max _{C}\left\{K_{2}\left(x, y^{0}\right)-K_{2}(x, y)\right\}=\psi(y) .
$$

We claim $\psi(y) \geqq 0$ for all $y$, for otherwise

$$
K_{2}\left(x, y^{0}\right)-K_{2}\left(x, y^{\prime}\right)<0 \text { for all } x \text { in } C \text { and for some } y^{\prime} \text {. }
$$

Thus $K_{2}\left(F^{0}, y^{0}\right)-K_{2}\left(F^{0}, y^{\prime}\right)<0$ contradicting $\left(F^{0}, G^{0}\right) \in \mathscr{E}$. Since $K_{2}(x$, $\left.y^{0}\right)-K_{2}(x, y)$ is convex for each $x$ and continuous in $x$ for each $y$ over the compact set $C$ it follows from the above proposition that for some $0 \leqq \lambda \leqq 1$

$$
\lambda K_{2}\left(x_{1}, y^{0}\right)+(1-\lambda) K_{2}\left(x_{2}, y^{0}\right) \geqq \lambda K_{2}\left(x_{1}, y\right)+(1-\lambda) K_{2}\left(x_{2}, y\right)
$$

for all $y$. Define $F^{*}=\lambda I_{x_{1}}+(1-\lambda) I_{x_{2}}$. Here $I_{x}$ stands for the degenerate distribution at $x$. Clearly $\left(F^{*}, y^{0}\right) \in \mathscr{E}$. The general case is handled by approximating $K_{2}(x, y)$ by a sequence of strictly concave functions in $y$. For the proof of Theorem 2 we need the following lemma of Glicksberg [2].

Lemma. Let $h(y) \geqq 0$ on the unit interval with $h^{n}(y)>0$. Then there exists a polynomial $p(y) \geqq 0$ of degree at most $(n-1)$ such that $h(y)-p(y) \geqq 0$ on $[0,1]$ and has exactly $n$ roots counting multiplicities.

For the sake of completeness we reproduce the proof.

Proof. By Rolle's theorem we know that $h(y)$ has at most $n$ roots. If it has exactly $n$ roots $p(y) \equiv 0$ is a choice. If $h(y)$ has 
fewer than $n$ roots, then $p(y)$ is constructed as follows. Let $y_{1}, y_{2}$, $\cdots, y_{k}$ be the roots of $h(y)$. Let $q(y)$ be a polynomial with the same roots and multiplicities. Then $q(y) \geqq 0$ and of degree at most $(n-1)$. Let $m_{i}$ be the multiplicity of $y_{i}$. Then

$$
\lim _{y \rightarrow y_{i}} \frac{h(y)}{q(y)}=\lim _{y \rightarrow y_{i}} \frac{h^{m_{i}}(y)}{q^{m_{i}}(y)}=\delta_{i}>0 .
$$

Hence we have open sets $E_{i}$ around $y_{i}$ with

$$
\frac{h(y)}{q(y)} \geqq \frac{\delta_{i}}{2} \quad \text { in } \quad E_{i} \text {. }
$$

The complement of $U E_{i}$ is a compact set, hence $h / q$ is the ratio of two continuous nonvanishing functions and that it achieves its minimum $\delta_{0}$. Hence for $\varepsilon<\min \left(\delta_{0}, \delta_{1} / 2\right) h(y)-\varepsilon q(y) \geqq 0$. Let $\varepsilon_{0}$ be the supremum of all $\varepsilon$ for which $h(y)-\varepsilon q(y) \geqq 0$. Then $h(y)-\varepsilon_{0} q(y)$ has at least one more root for otherwise $h(y)-\varepsilon_{0} q(y)$ would satisfy all the conditions that $h(y)$ satisfies and we cound find an $\varepsilon^{\prime}>0$ with

$$
\left[h(y)-\varepsilon_{0} q(y)\right]-\varepsilon^{\prime} q(y) \geqq 0
$$

which contradicts that $\varepsilon_{0}$ is the supremum. Therefore we either have a new root or at least the multiplicity of a former root is increased. The function $h-\varepsilon_{0} q \geqq 0$ satisfies

$$
\frac{\partial^{n}}{\partial y^{n}}(h-\varepsilon q)>0
$$

We may continue the process until we arrive at a $p(y)$ satisfying the conditions of the lemma. Further one easily checks that this polynomial is unique.

Proof of Theorem 2. First we prove the theorem when $K_{2}(x, y)$ is of the form $K_{2}(x, y)=\sum_{i=0}^{n} a_{i}(x) y^{i}$ with $\left(\partial^{n} / \partial y^{n}\right) K_{2}(x, y)<0$. Let $\left(F^{0}, G^{0}\right) \in \mathscr{E}$. Since $K_{2}\left(F^{0}, G^{0}\right)=\max K_{2}\left(F^{0}, y\right)$ and $\left(\partial^{n} / \partial y^{n}\right) K_{2}\left(F^{0}, y\right)<$ 0 we see that $\sigma\left(G^{0}\right)$ is finite. We will produce an $F^{*}$ with $\sigma\left(F^{*}\right)$ finite and $\left(F^{*}, G^{0}\right) \in \mathscr{E}$. Consider $S=\left\{\alpha=\left(\alpha_{0}, \alpha_{1}, \cdots, \alpha_{n}\right): \alpha_{i}=\int y^{i} d G, i=\right.$ $0,1,2, \cdots, n$, for some probability distribution $G\}$. Let $M(x, \alpha)=$ $\sum_{i} a_{i}(x) \alpha_{i}$. Clearly $S$ is compact and convex in $R^{n+1}$ and $M(x, \alpha)$ is affine in $\alpha$ for each $x$. Let $C=\sigma\left(F^{0}\right)$ and $K_{2}\left(x, G^{0}\right)=\sum a_{i}(x) \alpha_{i}^{0}=$ $M\left(x, \alpha^{0}\right)$.

Define $\psi(\alpha)=\max _{x \in C}\left\{M\left(x, \alpha^{0}\right)-M(x, \alpha)\right\}$. As in Theorem 1 we have $\psi(\alpha) \geqq 0$ for every $\alpha$ in $S$ and by the proposition of Bohnenblust, Karlin, and Shapley we have a finite number of points $x_{1}, x_{2}, \cdots, x_{m}$ in $C$ such that 


$$
\sum \lambda_{i} M\left(x_{i}, \alpha^{0}\right) \geqq \sum \lambda_{i} M\left(x_{i}, \alpha\right) \text { for all } \alpha \in S
$$

where $\lambda=\left(\lambda_{1}, \cdots, \lambda_{m}\right)$ is a probability vector. Let $F^{*}=\sum_{i} \lambda_{i} I_{x_{i}}$. Clearly $\left(F^{*}, G^{0}\right) \in \mathscr{E}$. This proves Theorem 2 for the special case.

Proof of the theorem in the general case. Let $\left(F^{0}, G^{0}\right) \in \mathscr{E}$ and $h(y)=\int K_{2}(x, y) d F^{0}(x)$. Without loss of generality $K_{2}\left(F^{0}, G^{0}\right)=0$, and hence $h(y) \leqq 0$ for all $y$. From the above lemma of Glicksberg we have a polynomial $P(y)$ with $h(y) \leqq P(y) \leqq 0$ and $P(y)-h(y)$ has exactly $n$ roots counting multiplicities. For each $x$ we can find coefficients $a_{i}(x), i=0,1, \cdots, n-1$ with $\sum a_{i}(x) y^{i}-K_{2}(x, y)$ having the same roots and multiplicities as that of $p(y)-h(y)$.

Now we will show that the coefficients $a_{i}(x)$ are continuous in $x$, for $i=0,1,2, \cdots, n-1$. Fix $x=x_{0}$ and consider $\sum_{i=0}^{n-1} a_{i}\left(x_{0}\right) y^{i}-$ $K_{2}\left(x_{0}, y\right)$. The polynomial $\sum_{i=0} a_{i}\left(x_{0}\right) y^{i}$ is unique for otherwise we will have 2 distinct polynomials of degree less than $n$ whose difference will have $n$ roots counting multiplicities. This is clearly not possible. Thus for each fixed $x$ the coefficients $a_{i}(x)$ are uniquely determined. In fact we may write $n$ linear equations for the unknown $a_{0}(x), \cdots$, $a_{n-1}(x)$. The matrix of coefficients in these equations is nonsingular and since this matrix is independent of $x$, it follows from the continuity of $K_{2}(x, y)$ in $x$, that the $a_{i}(x)$ 's are continuous in $x$. Now since the roots interior to $[0,1]$ are of even multiplicity

$$
\sum a_{i}(x) y^{i}-K_{2}(x, y) \geqq 0 \text { for all }(x, y)
$$

or

$$
\sum a_{i}(x) y^{i}-K_{2}(x, y) \leqq 0 \text { for all }(x, y) \text {. }
$$

But $\int \sum a_{i}(x) y^{i} d F^{0}(x)-K_{2}\left(F^{0}, y\right)$ has roots and multiplicities as that of $P(y)-h(y)$. Since $P(y)$ is unique $P(y)=\int \sum a_{i}(x) y^{i} d F^{0}(x)$. Since $P(y)>h(y)$ for some $y$ the first inequality holds.

Since $K_{2}(x, y) \leqq \sum a_{i}(x) y^{i}=P(x, y)$

$$
\begin{aligned}
0=K_{2}\left(F^{0}, G^{0}\right) & \leqq \iint \sum a_{i}(x) d F^{0}(x) y^{i} d G^{0}(y)=P\left(F^{0}, G^{0}\right) \\
& \leqq \int P(y) d G^{0}(y) \leqq 0
\end{aligned}
$$

Thus $P\left(F^{0}, G^{0}\right)=0 \geqq P\left(F^{0}, y\right)=P(y)$ for all $y$. We therefore have $\left(F^{0}, G^{0}\right)$ as an equilibrium point of the auxiliary game. We know from the first part that this can be replaced by $\left(F^{*}, G^{0}\right)$ where $\sigma\left(F^{*}\right)$ is a finite subset of $\sigma\left(F^{0}\right)$. We claim $\left(F^{*}, G^{0}\right)$ is also an equilibrium point for the original game. To show this it is enough to prove $K_{2}\left(F^{*}, G^{0}\right)=P\left(F^{*}, G^{0}\right)$ for then $K_{2}\left(F^{*}, G^{0}\right)=P\left(F^{*}, G^{0}\right) \geqq$ 
$P\left(F^{*}, G\right) \geqq K_{2}\left(F^{*}, G\right)$ for all $G$. Suppose $K_{2}\left(F^{*}, G^{0}\right)<P\left(F^{*}, G^{0}\right)$ (the other inequality cannot hold). Then $K_{2}(x, y)<P(x, y)$ for some $x \in \sigma\left(F^{*}\right) \subset \sigma\left(F^{0}\right)$ and $y \in \sigma\left(G^{0}\right)$ and $K_{2}(x, y) \leqq P(x, y)$ for all $(x, y)$. Combining these two statements we have $0=K_{2}\left(F^{0}, G^{0}\right)<P\left(F^{0}, G^{0}\right) \leqq$ 0 a contradiction. Hence the assertion. This completes the proof of Theorem 2.

3. In this section we give a set of sufficient conditions for the uniqueness of optimal strategies and equilibrium points for continuous games.

Definition. Let $\mu_{1}, \mu_{2}$ be two probability measures on the unit interval. We say $\mu_{1} \leqq \mu_{2}$ if for some $k>0 \mu_{1}(E) \leqq k \mu_{2}(E)$ for all $E$. We say $\mu_{1} \sim \mu_{2}$ if $\mu_{1} \leqq \mu_{2}$ and $\mu_{2} \leqq \mu_{1}$.

THEOREM 3. Let $K(x, y)$ be a continuous payoff on $0 \leqq x, y \leqq 1$ for a zero-sum two-person game. Suppose every optimal strategy for each player has the spectrum the entire unit interval. Further assume every pair of optimal strategies for each player be equivalent. Then there is only one optimal strategy for each player.

THEOREM 4. Let $K_{1}(x, y), K_{2}(x, y)$ be continuous payoffs on $0 \leqq$ $x, y \leqq 1$ for a nonzero-sum two-person game. Let for every $\left(\mu_{1}, \nu_{1}\right) \in$ $\mathscr{E},\left(\mu_{2}, \nu_{2}\right) \in \mathscr{E} \sigma\left(\mu_{1}\right)=\sigma\left(\mu_{2}\right)=\sigma\left(\nu_{1}\right)=\sigma\left(\nu_{2}\right)=[0,1]$ and $\mu_{1} \sim \mu_{2}, \nu_{1} \sim \nu_{2}$. Then $\mathscr{E}$ has just one element.

Proof of Theorem 3. It suffices to prove that the compact convex set of optimal strategies for each player has exactly one extreme point.

Let if possible $\mu_{1}, \mu_{2}$ be two distinct extreme optimal strategies, say for player I. Since $\mu_{1} \sim \mu_{2}$ we have a $k>2$ such that $\mu_{1}(E) \leqq$ $k \mu_{2}(E)$ for all $E$. Define

$$
\mu^{\prime}(E)=(1+\theta) \mu_{2}(E)-\theta \mu_{1}(E)
$$

and

$$
\mu^{\prime \prime}(E)=(1-\theta) \mu_{2}(E)+\theta \mu_{1}(E)
$$

where

$$
0<\theta=\frac{1}{k-1}<1 .
$$

When the spectrum of an optimal strategy for player II is the whole unit interval every optimal strategy for player $I$ is an equalizer. That is 


$$
\int K(x, y) d \mu_{1}(x) \equiv v \quad \text { for all } \quad y
$$

and

$$
\int K(x, y) d \mu_{2}(x) \equiv v \text { for all } y
$$

Thus it is easily seen that $\mu^{\prime}, \mu^{\prime \prime}$ are two distinct optimal strategies for player I and that $\mu_{2}=\left(\mu^{\prime}+\mu^{\prime \prime}\right) / 2$. This contradicts the fact that $\mu_{2}$ is an extreme optimal for player I. Hence the theorem.

Proof of Theorem 4. Since for any pair $\left(\mu_{1}, \nu_{1}\right) \in \mathscr{E},\left(\mu_{2}, \nu_{2}\right) \in \mathscr{E}$ we have

$$
\begin{aligned}
& \int K_{1}(x, y) d \nu_{2}(y) \equiv c_{2}, \int K_{2}(x, y) d \mu_{1}(x) \equiv \alpha_{1} \\
& \int K_{1}(x, y) d \nu_{1}(y) \equiv c_{1}, \int K_{2}(x, y) d \mu_{2}(x) \equiv \alpha_{2}
\end{aligned}
$$

$\left(\mu_{1}, \nu_{2}\right)$ and $\left(\mu_{2}, \nu_{1}\right)$ also belong to $\mathscr{E}$ and that $\mathscr{E}$ is a convex set. Of course $\mathscr{E}$ is compact. The rest of the proof is as in Theorem 3.

REMARK 1. For example if every optimal strategy for each player possesses a continuous strictly positive density, then one can check that the conditions of Theorem 3 are satisfied; hence such games will have unique optimal strategies.

REMARK 2. For matrix games the notion of equivalence coincides with the notion of completely mixed strategies.

REMARK 3. It would be interesting to know whether Theorem 3 is valid if we just assume that the spectrum of every optimal strategy for each player is the unit interval.

ACKNowledment. We wish to thank the referee for some useful comments.

\section{REFERENCES}

1. H. F. Bohnenblust, S. Karlin, and L. S. Shapley, Games with continuous convex payoff, Annals of Mathematics Studies, 24 edited by H. W. Huhn and A. W. Tucker, Princeton University Press, (1950), 181-192.

2. I. Glicksberg, $A$ derivative test for finite solutions of games, Proc. Amer. Math. Soc., 4 (1953), 895-897.

3. T. Parthasarathy and T. E. S. Raghavan, Some Topics in Two Person Games, Amer. Elsevier Publ. Co., New York, 1971.

Received February 27, 1974. The first author is on leave from the Indian Statistical Institute, Calcutta. 


\title{
PACIFIC JOURNAL OF MATHEMATICS
}

\author{
EDITORS
}

RICHARD ARENS (Managing Editor)

University of California

Los Angeles, California 90024

R. A. Beaumont

University of Washington

Seattle, Washington 98105
J. DugundJI

Department of Mathematics University of Southern California Los Angeles, California 90007

D. Gilbarg and J. Milgram

Stanford University

Stanford, California 94305

\section{ASSOCIATE EDITORS}

E. F. BECKENBACH

B. H. NeUManN

F. WOLF

K. YosHIDA

\section{SUPPORTING INSTITUTIONS}

UNIVERSITY OF BRITISH COLUMBIA

GALIFORNIA INSTITUTE OF TECHNOLOGY

UNIVERSITY OF CALIFORNIA

MONTANA STATE UNIVERSITY

UNIVERSITY OF NEVADA

NEW MEXICO STATE UNIVERSITY

OREGON STATE UNIVERSITY

UNIVERSITY OF OREGON

OSAKA UNIVERSITY
UNIVERSITY OF SOUTHERN CALIFORNIA

STANFORD UNIVERSITY

UNIVERSITY OF TOKYO

UNIVERSITY OF UTAH

WASHINGTON STATE UNIVERSITY

UNIVERSITY OF WASHINGTON

AMERICAN MATHEMATICAL SOCIETY NAVAL WEAPONS CENTER 


\section{Pacific Journal of Mathematics}

\section{Vol. 57, No. $1 \quad$ January, 1975}

Keith Roy Allen, Dendritic compactification ......................... 1

Daniel D. Anderson, The Krull intersection theorem ..................... 11

George Phillip Barker and David Hilding Carlson, Cones of diagonally dominant

matrices .............................................

David Wilmot Barnette, Generalized combinatorial cells and facet splitting ........

Stefan Bergman, Bounds for distortion in pseudoconformal mappings ............

Nguyên Phuong Các, On bounded solutions of a strongly nonlinear elliptic

equation ...............................................

Philip Throop Church and James Timourian, Maps with 0-dimensional critical

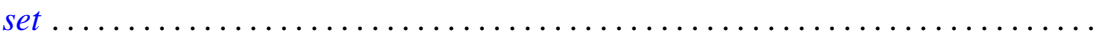

G. Coquet and J. C. Dupin, Sur les convexes ubiquitaires ................. 67

Kandiah Dayanithy, On perturbation of differential operators............... 85

Thomas P. Dence, A Lebesgue decomposition for vector valued additive set

functions .............................................. 91

John Riley Durbin, On locally compact wreath products.................. 99

Allan L. Edelson, The converse to a theorem of Conner and Floyd ............. 109

William Alan Feldman and James Franklin Porter, Compact convergence and the

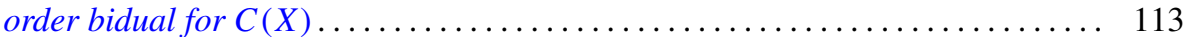

Ralph S. Freese, Ideal lattices of lattices......................... 125

R. Gow, Groups whose irreducible character degrees are ordered by divisibility ... 135

David G. Green, The lattice of congruences on an inverse semigroup ............ 141

John William Green, Completion and semicompletion of Moore spaces .......... 153

David James Hallenbeck, Convex hulls and extreme points of families of starlike and close-to-convex mappings .................................. 167

Israel (Yitzchak) Nathan Herstein, On a theorem of Brauer-Cartan-Hua type ...... 177

Virgil Dwight House, Jr., Countable products of generalized countably compact spaces ............................................ 183

John Sollion Hsia, Spinor norms of local integral rotations. I . . . . . . ......... 199

Hugo Junghenn, Almost periodic compactifications of transformation

semigroups....................................

Shin'ichi Kinoshita, On elementary ideals of projective planes in the 4-sphere and oriented $\Theta$-curves in the 3 -sphere ............................ 217

Ronald Fred Levy, Showering spaces............................ 223

Geoffrey Mason, Two theorems on groups of characteristic 2-type............. 233

Cyril Nasim, An inversion formula for Hankel transform ................... 255

W. P. Novinger, Real parts of uniform algebras on the circle ................ 259

T. Parthasarathy and T. E. S. Raghavan, Equilibria of continuous two-person

games............................................... 265

John Pfaltzgraff and Ted Joe Suffridge, Close-to-starlike holomorphic functions of several variables ....................................... 271

Esther Portnoy, Developable surfaces in hyperbolic space .................. 281

Maxwell Alexander Rosenlicht, Differential extension fields of exponential type . . . 289

Keith William Schrader and James Lewis Thornburg, Sufficient conditions for the

existence of convergent subsequences ........................... 301

Joseph M. Weinstein, Reconstructing colored graphs .................. 307 